\title{
Interference in letter identification: A test of feature-specific inhibition
}

\author{
JEFFREY L. SANTEE and HOWARD E. EGETH \\ The Johns Hopkins University, Baltimore, Maryland 21218
}

\begin{abstract}
The role of perceptual interference in letter identification was investigated in three experiments designed to test the feature-specific inhibition model proposed by Bjork and Murray (1977). According to their extension of Estes' $(1972,1974)$ interactive channels model, input channels leading to the same feature detector inhibit one another more than do channels leading to different detectors. The model therefore predicts perceptual interference between two letters to be a function of the degree of their feature overlap. Experiment 1 confirmed the feature-specific inhibition model and Bjork and Murray's finding that the accuracy of report is lower when a briefly presented target letter is flanked by an identical letter than when flanked by another target letter or by a nontarget letter. Results from Experiment 2 indicated that single-target performance is a function of the degree of feature similarity between the target letter and background characters in a stimulus display. Experiment 3 ruled out a spatial-uncertainty explanation of feature-specific inhibition in a new paradigm that does not require subjects to process a poststimulus cue. The results of these experiments are discussed in relation to recent studies exhibiting strong effects of noise letters at the response stage of processing. It is suggested that discrepancies between feature-specific interference and response-interference studies may be a function of the particular mode of stimulus presentation and of the dependent measures that are used.
\end{abstract}

Bjork and Murray (1977) have recently proposed a model of visual processing that emphasizes featurespecific inhibition among visual input channels. The model is based upon their finding that the accuracy of report is lower when a briefly presented target letter (e.g., B) is flanked by an identical letter than when flanked by another target letter (e.g., R) or by a nontarget letter (e.g., $P$ or K). For instance, they found that if a poststimulus cue indicated that the left-hand member of a letter pair was to be reported, then report of $B$ from the pair BB was less accurate than report of B from the pair BR or from the pair BK.

The feature-specific inhibition model, as proposed by Bjork and Murray, assumes that alphanumeric characters are represented in memory as hierarchical lists of features (e.g., Estes, 1972; Rumelhart, 1970; Wolford, 1975). Information about the features contained within visually presented stimuli is extracted over parallel, but interactive, input channels that lead to feature detectors. According to the model, the excitation of a particular input channel caused by the presence of a particular feature contained within a letter results in both feature-specific inhibition of other

This research was supported in part by a grant from the National Science Foundation (BNS-76-01227) to Howard Egeth and James Pomerantz. The authors would like to thank Alfonso Caramazza, Charles W. Eriksen, Michael McCloskey, James Pomerantz, Lawrence Sager, and Edward E. Smith for their helpful advice. Requests for reprints should be sent to either Jeffrey Santee or Howard Egeth, Department of Psychology, The Johns Hopkins University, Baltimore, Maryland 21218. channels leading to the same feature detector and a more generalized inhibition of all input channels (cf. Estes, 1972). Thus, the feature-specific inhibition model predicts perceptual interference between two letters to be a function of the degree of their feature overlap. This is consistent with Bjork and Murray's rather counterintuitive finding that the accuracy of report of a cued letter is lower when the letter is flanked by an identical letter than when flanked by another letter.

This result is surprising in view of the fact that in most other paradigms stimulus repetitions have been shown to facilitate performance. For example, in a choice reaction time paradigm, subjects typically respond faster on trials involving repetition of the stimulus from the preceding trial than on trials involving nonrepetition (e.g., Bertelson, 1961; Felfoldy, 1974). In lexical decision tasks, subjects respond more quickly when a stimulus is primed by a semantically related word than when it is preceded by an unrelated word (see, e.g., Schvaneveldt \& Meyer, 1973). Also, when speeded same-different judgments are required, responses are often faster to same than to different pairs (e.g., Bamber, 1969; Egeth \& Blecker, 1971; Krueger, 1978). More importantly, within the realm of letter perception itself, target redundancy has been shown to improve recognition accuracy (Eriksen, 1966; Eriksen \& Lappin, 1965).

The major interest in the feature-specific inhibition model derives from the fact that predictions from the model are inconsistent with other major theoretical 
accounts of tachistoscopic letter perception. Specifically, the prediction that the perception of, say, the letter B on the left in the pair BB would be worse than in the pair $B R$ is inconsistent with the major predictions of the independent channels model (see Gardner, 1973; Kinchla, 1974; Shiffrin \& Geisler, 1973) and the continuous flow model based on the idea of response competition (Eriksen \& Eriksen, 1974, 1979; Eriksen \& Schultz, 1979). According to these "postperceptual" models, information is extracted over independent, parallel channels, and interference between target and noise letters occurs at either a decision or response selection stage of processing. Therefore, letter processing should not be a function of interactions among input channels during the early perceptual stages of processing, but should result from confusions at a subsequent decision or response level over which letter is the target element in a display. Estes' $(1972,1974)$ interactive channels model is similar to the featurespecific inhibition model. Both models are "perceptual" in that the effects of noise elements on decisionlevel processes are considered to be secondary to the interference between target and noise elements resulting from the interaction of visual input channels during the initial stages of feature extraction. However, Estes' model, which posits only a general inhibition among input channels, fails to account for Bjork and Murray's chief results. (For a more detailed discussion of the major models of letter perception and their primary predictions, the reader is referred to Bjork \& Murray, 1977).

In the present paper, our chief concern was to provide an independent test of the feature-specific inhibition model. We believe that Bjork and Murray's (1977) major finding deserved replication for two basic reasons. First, the feature-specific inhibition model was developed post hoc and was based on the results of one study with only one set of letters. It is possible that their data, although interesting, may have resulted from the fortuitous selection of a peculiar set of letters. To the extent that this is true, the model rests on a relatively weak foundation. Second, since the results reported by Bjork and Murray stand in direct conflict with the predictions from most other models of letter perception, plausible alternative explanations of their data need to be tested.

The purpose of Experiment 1 was to first verify the existence of feature-specific inhibition among input channels at the perceptual level with a set of target and noise letters different from those employed by Bjork and Murray. In addition, the experiment sought to nvestigate inhibitory effects with foveated stimuli and relatively focused attenticn by presenting the target and noise letters in only two possible locations. Experiment 2 investigated the role that background characters in a stimulus display can play in the detection of target etters. Finally, Experiment 3 investigated the role of terture-specific inhibition in a paradigm that did not equire $\mathrm{s}$ lbjects to process a poststimulus cue.

\section{EXPERIMENT 1}

Until recently, it has been difficult to discriminate empirically between the perceptual and postperceptual models described earlier because both types of models predict a decrease in detection accuracy with corresponding increases in the number of noise elements and the level of target-noise confusability, due to interactions at the decision level. Therefore, in order to test for the inhibitory effects of noise elements at the perceptual level, the effects of noise elements at the decision level should be minimized. Bjork and Murray have reported the use of a forced-choice detection paradigm which minimizes the effects of noise items at the decision level by cuing only target elements.

Experiment 1 adopted the paradigm used by Bjork and Murray with a few modifications. The same sequence of events occurred on each trial. First, a premask of dollar signs (\$) arranged in a 3 (row) by 4 (column) matrix was presented for $1 \mathrm{sec}$, followed immediately by one of several possible types of stimulus displays. Either one or both of the middle two positions of the 3 by 4 matrix in each stimulus display contained a letter. The remaining positions were filled with symbols resembling number signs (\#). A postmask containing a 3 by 4 matrix of backward dollar signs immediately followed the stimulus array. At the bottom of the postmask was an upward pointing arrow directly under one of the middle two columns. The subject's task was to determine which of the two target letters, A or E, was present in the cued column of the stimulus display.

Figure 1 provides examples of the four types of conditions used in Experiment 1. The nomenclature for these conditions has been borrowed from Bjork and Murray. The correct response to each of the example displays would be " $A$ " if the left of the middle two columns were cued. In the single-target condition (A\#), only a single target letter was presented and cued. The three double-letter displays, however, contained both target and noise letters and differed with respect to the type of noise letters presented. In the noise-same-as-target condition (AA), the two letters were physically identical but only the cued letter served as the target. Unlike many detection paradigms, this permitted the uncued letter to act as a potentially interfering noise item rather than as a potentially facilitating redundant signal, thus allowing for the separation of target-noise similarity and confusability. In the noise-alternative-target condition (AE), both of the target letters were presented. Again, only one of the letters was cued. Finally, the noise-nontarget condition (AK) contained a target letter in the presence of one of the two nontarget letters $\mathrm{K}$ or $\mathrm{L}$.

Assuming that the poststimulus cue is effective, the paradigm described above permits a high degree of target noise similarity without confusion over which letter is the target and which is noise in the AA condi- 


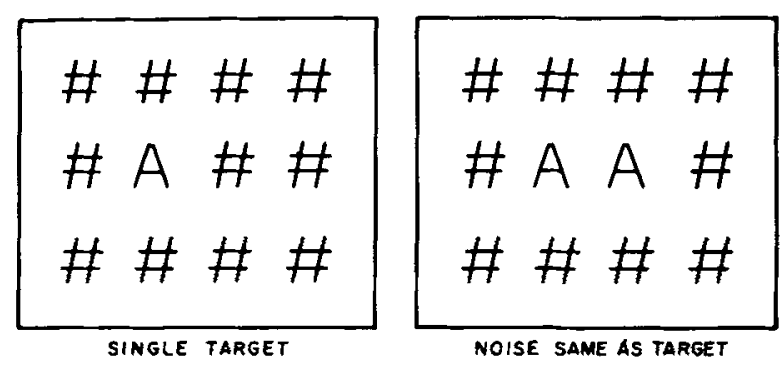

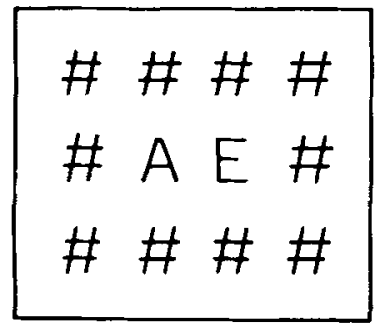

NOISE ALTERNATIVE TARGET

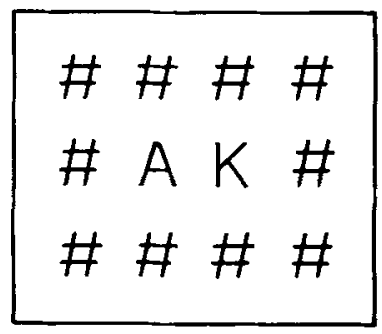

MOISE NONTARGET
Figure 1. An example of the four display conditions in Experiment 1 . In each of the example displays, the target letter $A$ would be cued in the left of the middle two columns.

tion. According to the feature-specific inhibition model, input channels leading to feature detectors of both target letters are assumed to be in a state of heightened excitability due to the subjects' instructional set (Estes, 1972). However, since channels leading to the same feature detectors inhibit each other more than channels going to other detectors, mutual inhibition between two elements should be a function of the degree to which those elements have the same features. This means that the poorest performance should be found in the AA condition. The AE and AK conditions should generate approximately equal levels of performance, because the degree of feature overlap among the target and nontarget letters is approximately the same in both conditions (see Townsend's 1971, interletter confusion matrices). The model is also consistent with finding the best performance in the A\# condition, provided the target letters do not share too many features with the number-sign character.

\section{Method}

Subjects. Twelve undergraduates at Johns Hopkins University served as paid subjects. All had normal or corrected-to-normal vision. Each was run individually for two sessions on 2 consecutive days. The preliminary session lasted about $30 \mathrm{~min}$, and the experimental session lasted $1 \mathrm{~h}$.

Apparatus. All displays were presented in a four-channel ICONIX tachistoscope automated by a solid-state logic system. Stimulus materials were typed on cards using an IBM Selectric typewriter with an Artisan-12 element. Premask, stimulus, and postmask displays each contained a 3 (row) by 4 (column) matrix of symbols centered in the visual field at a viewing distance of $91 \mathrm{~cm}$. The height and width of each matrix subtended visual angles of 1.01 and 1.13 deg, respectively. Each character was $.3 \mathrm{~cm}$ high and $.2 \mathrm{~cm}$ wide, and there was a space of $.4 \mathrm{~cm}$ between adjacent rows and columns. The overall angle subtended by the middle two characters was $.5 \mathrm{deg}$. The premask matrix contained dollars signs (\$). Each of the two postmasks was composed of backward dollar signs with an upward-pointing arrow positioned below one of the middle two columns. In each stimulus display, one or both of the middle two positions were filled with letters. The remaining positions were occupied by number-sign characters (\#).

Procedure. The target letters $\mathrm{A}$ and $\mathrm{E}$ appeared equally often in each of the four stimulus conditions and as the cued letters in each of the middle two positions. Also, the noise letters $\mathrm{K}$ and $\mathrm{L}$ appeared equally often with each of the target letters in the AK condition. The 576 experimental trials were divided equally among the four stimulus conditions. Displays were presented in 18 blocks of 32 trials each during the experimental session. Within each block, both target letters and each of the four types of conditions appeared equally often and in a randomized order. Each of the middle two columns was also cued an equal number of times within each block of trials. Subjects responded by pressing either a top button with their index fingers or a bottom button with their thumbs, while making a verbal response. 'The two target-response assignments were counterbalanced across subjects.

During the preliminary session, the exposure duration of the stimulus displays was adjusted until each subject performed between $75 \%$ and $80 \%$ accuracy, averaged across all conditions for three blocks of trials. Duration for individual subjects ranged from 75 to $35 \mathrm{msec}$; this was adjusted at the end of each block of trials in order to maintain $75 \%$ to $80 \%$ accuracy throughout the experimental session. At the beginning of each session, the subjects were told that either an $A$ or an $E$ would be cued on each trial. They were also informed that the two target letters as well as the left and right columns would be cued with equal probability no matter what appeared in the uncued columns. Responses were to be based on only the information that was present in the cued column. In other words, they were informed that information in the uncued column would not provide them with any information about the letter present in the cued column. The subjects were told that the experimenter was interested only in how accurately they could recognize the letters, not in how quickly they could respond. They were encouraged, however, to make a reasonably prompt response even though reaction time was not recorded.

The first trial in each block was initiated by the experimenter. Each trial began with a 1-sec premask display which served as a ready signal. The subjects were instructed to focus on the middle two positions of the matrix during this period. Following the premask, a stimulus display appeared and was followed immediately by a postmask containing an upward-pointing arrow underneath one of the middle two columns. The postmask and cue stayed in view until the subject made a response, at which time they were replaced by the premask. After the subject made a response, he initiated the next trial by pressing a button. The new trial began approximately $2.5 \mathrm{sec}$ later. A 5 -min rest period was given midway through the experimental session.

\section{Results and Discussion}

Table 1 presents the mean proportions of correct detections of both target letters in each of the four conditions. Data averaged across both target letters are also presented and compared to that found by Bjork and Murray (1977), in which the same four conditions were used with a different set of letters. An analysis of variance performed on our data revealed a significant main effect of display conditions $[F(3,33)$ $=7.34, p<.001]$. Neither the main effect of target 
Table 1

Mean Proportions of Correct Detections of Both Target Letters in Each Display Condition of Experiment 1

\begin{tabular}{lcccc}
\hline & \multicolumn{4}{c}{ Target Letters } \\
\cline { 2 - 5 } \multicolumn{1}{c}{ Display Conditions } & A & E & \multicolumn{2}{c}{ Pooled } \\
\hline Single Target (A\#) & .79 & .74 & .76 & $(.90)$ \\
Noise Same as Target (AA) & .72 & .72 & .72 & $(.74)$ \\
Noise Alternative Target (AE) & .84 & .84 & .84 & $(.84)$ \\
Noise Nontarget (AK) & .85 & .86 & .86 & $(.82)$ \\
\hline
\end{tabular}

Note-Bjork and Murray (1977) results are shown in parentheses.

letter nor the Target Letter by Display Condition interaction attained significance.

Upon inspection, it is apparent that the pattern of results obtained in this experiment is in close agreement with Bjork and Murray's (1977) results and the predictions of the feature-specific inhibition model. Specifically, performance was significantly lower in the AA condition than in either the AE or the AK conditions $[\mathrm{t}(11)=3.33, \mathrm{p}<.01$, and $\mathrm{t}(11)=3.98, \mathrm{p}<.01$, respectively]. ${ }^{2}$ Virtually the same level of performance was attained in the latter two conditions.

There is one aspect of the data, however, that appears not to conform to the predictions of the feature-specific inhibition model. Namely, the highest level of performance was not obtained in the A\# condition. As may be seen in Table 1, performance in our A\# condition was considerably lower than that observed by Bjork and Murray. This particular issue will be addressed in Experiment 2.

The low level of performance in the AA condition relative to performance in the other two double-letter conditions provides support for the feature-specific inhibition model. Furthermore, the model has been shown to predict performance with a set of letters different from those used by Bjork and Murray and in a situation in which the subject's attention has been directed to two specific, adjacent locations. In the Bjork and Murray study, the target letters appeared in all possible locations of 4 by 4 matrix display. Our results indicate that a considerable amount of interaction between input channels takes place even when visual images fall on or near the fovea.

Response bias. It might appear that the chief results of the Bjork and Murray study and this experiment could be explained in terms of response bias. To see how this might be done, let us turn our attention to the crucial difference between the AA and AE conditions, and make a few plausible assumptions.

First, assume that on some trials one of the letters in the double-letter displays is not seen clearly, and that on approximately half of these trials the unclear letter is in the cued column. Second, assume that the subject has a bias to respond to a cued but undifferentiated letter with the name of the target letter opposite that of the letter seen clearly. For example, if the subject sees an $\mathbf{A}$ and a cued but unclear letter, then he or she would tend to respond "E." It follows that accuracy would be lower when the noise (i.e., uncued) letter is the same as the target (as in the AA condition) than when the noise letter is the alternative target (as in the AE condition). Thus, performance differences in the AA and AE conditions might be an artifact of this response bias rather than the result of perceptual interference.

Bjork and Murray considered a similar response-bias hypothesis to be implausible. We also believe that it is implausible for a number of reasons. First, subjects were completely informed that the uncued letters would not provide them with any information concerning the identity of the cued letter. Second, the AK condition may be viewed as a built-in control for response bias. That is, to the extent that the results of Experiment 1 may be attributed to response bias, performance should be higher in the $\mathrm{AE}$ than in the AK condition. As noted earlier, the same level of performance was obtained in these two conditions.

Finally, we have conducted two experiments to test the response-bias hypothesis. In one experiment, performance in the $\mathrm{AA}$ and $\mathrm{AE}$ conditions was measured along with performance in a third condition in which a target letter appeared with the nontarget letter O (e.g., AO, EO). In order to force subjects to view both the nontarget letter and the flanking target letter in the latter condition, each was cued with equal probability. Whenever the nontarget letter $O$ was cued, the subjects were required to make either an $\mathrm{A}$ or an $E$ response based on whatever they thought was the most appropriate response on that trial. An identical experiment was conducted on a different set of subjects, except that ambiguous characters were used in place of the nontarget letter $O$. One of the ambiguous characters consisted of an uppercase $A$ and an uppercase $\mathrm{E}$ superimposed by overstriking. The other ambiguous character consisted of an $\mathrm{A}$, an $\mathrm{E}$, and a \#, all three elements superimposed by overstriking.

The response-bias hypothesis, as outlined above, predicts that subjects would tend to respond to a cued nontarget character with the name of the target letter not present on that trial. Neither experiment provided evidence to support this prediction. If anything, there was a slight tendency for subjects to respond with the name of the letter that was the same as the flanking target letter. Furthermore, performance was significantly lower in the AA condition than in the AE condition in both experiments. These results suggest that response bias cannot account for the results obtained in Experiment 1 or in Bjork and Murray's study.

\section{EXPERIMENT 2}

One troublesome aspect of Experiment 1 that needs to be addressed is the poor performance we observed in the A\# condition. According to Bjork and Murray's explication of feature-specific inhibition, the highest level of performance should have been obtained in the 
A\# condition because the inhibitory effects of input channels leading to feature detectors associated with the number-sign characters would be lower than the inhibitory effects of input channels leading to detectors associated with the target letters. This prediction follows from two basic assumptions: (1) that input channels leading to detectors necessary for the detection of target letters will be in a high state of excitability due to a subject's readiness to respond to target letters, and (2) that the amount of inhibition exerted by a detector's input channel is proportional to its state of excitability (Estes, 1972). It should be noted, however, that the prediction of better performance in the A\# condition depends critically on the (implicit) assumption that the target and background characters share few features.

We think that the low level of accuracy in the A\# condition of Experiment 1 is not necessarily inconsistent with the feature-specific inhibition model. In fact, it could readily be explained if there were a greater degree of feature overlap between the target letters and the number-sign characters used in this study compared to those used by Bjork and Murray.

To test this explanation, the target letters from our Experiment 1 ( $\mathrm{A}$ and $\mathrm{E}$ ) and the target letters from the Bjork and Murray study (B and R) were used in the single-target condition embedded in matrices consisting of number-sign characters (\#). This provided a replication of the single-target condition from our first experiment and Bjork and Murray's experiment. We expected to replicate the respective results as well; the A vs. E decision ought to yield poorer performance than the $B$ vs. $R$ decision. Of course, this might only mean that $A$ vs. $E$ is inherently more difficult than $B$ vs. $R$, while our speculation is concerned with targetbackground featural similarity. Therefore, to provide a more discriminating test, another background character was also used - the letter P. This letter was chosen because it appears more similar to $\mathbf{B}$ and $\mathbf{R}$ than to $\mathrm{A}$ and $\mathrm{E}$. Consequently, we expected that the $A$ vs. $E$ decision would be easier than the $B$ vs. $R$ decision when stimulus matrices contained repeated $P s$ as background elements. In other words, an interaction between target-letter set (i.e., $\mathbf{A}$ and $\mathrm{E}$ vs. $\mathrm{B}$ and $\mathrm{R}$ ) and type of background character was expected.

\section{Method}

Subjects. Twelve undergraduates at Johns Hopkins University served as paid subjects. Each was run individually for one experimental session lasting approximately $1 \mathrm{~h}$. None of the subjects had served in Experiment 1. All had normal or corrected-to-normal vision.

Apparatus and Procedure. The stimulus materials and procedure were the same as in Experiment 1, with a few minor exceptions specified below. Performance in only the single-target condition was investigated. Six subjects responded to the target letters $A$ and $E$, while the targets for the remaining six subjects were $B$ and $R$. Within each of the target-letter sets, all matrix positions, except where a target letter appeared, were filled with either the numbersign character (\#) or the letter P. The two types of background characters never appeared together.
At the beginning of the experimental session, the exposure duration of the stimulus displays was adjusted until the subject reached $75 \%$ to $80 \%$ accuracy, averaged across both types of noise background for six blocks of trials. Within each block of 24 trials, the backgrounds were presented in 2 sets of 12 consecutive trials each. Once established, the duration for each subject was held constant throughout the remainder of the experimental session. The mean duration of approximately $50 \mathrm{msec}$ was comparable to that obtained in Experiment 1.

Following the preliminary trials, 10 blocks of 24 trials each were presented. For both groups of subjects, the number-sign background and the $P$ background were each used for five blocks of trials (i.e., 120 trials each). The type of background presented in the first two blocks of trials was counterbalanced across the six subjects in each group. The remaining eight blocks, four containing the number-sign background and four composed of the $P$ background, were presented in a random order. Within each block of 24 trials, both target letters appeared equally often in the middle two matrix positions, and in randomized order. Each of the middle two columns was cued an equal number of times. Both groups of subjects were given the same general instructions as provided in Experiment 1.

\section{Results and Discussion}

Presented in Table 2 are the mean proportions of correct detections obtained in each target set and background condition. Comparable values in the number-sign background condition that were obtained in Experiment 1 and by Bjork and Murray (1977) are also provided. Our primary interest was in the nature of the interaction between the target sets and background conditions, irrespective of individual targetletter performance. A preliminary analysis within each target set revealed a significant main effect of background in both sets $(\mathrm{p}<.01)$, but failed to show a significant interaction between individual target letters and background characters in either set (p > .10).

Therefore, the data, as shown in Table 2, were collapsed across the target letters in each set, and the resulting scores were subjected to a three-way analysis of variance (Target Sets by Backgrounds by Subjects). As expected, the main effects of backgrounds and target sets did not attain significance (Fs $<1)$. This is due to the fact that the exposure durations were adjusted during the preliminary trials so that $75 \%$ to $80 \%$ accuracy would be obtained across the two background conditions of the sets. The Target Sets by Backgrounds interaction, however, was highly significant $[\mathrm{F}(1,10)=134.48, \mathrm{p}<.001]$.

The nature of this interaction can be seen in Table 2 .

Table 2

Mean Proportions of Correct Detections in each Target and Background Condition of Experiment 2

\begin{tabular}{ccc} 
& \multicolumn{2}{c}{ Target } \\
\cline { 2 - 3 } Background & $\mathrm{A}$ and E & B and $\mathrm{R}$ \\
\hline$\#$ & $.73^{*}$ & $.88 \dagger$ \\
$\mathrm{P}$ & .85 & .73 \\
\hline
\end{tabular}

*Result from Experiment $1=.76$.

†Result from Bjork and Murray (1977) $=.90$. 
A lower level of performance was obtained in the number-sign background condition when the target letters were $\mathrm{A}$ and $\mathrm{E}$ compared to when the targets were $B$ and $R$. This result provides clear support for our argument that the level of performance in the A\# condition reflects the similarity between the specific targets and noise characters that are used. McIntyre, Fox, and Neale (1970) obtained a similar pattern of results while using the letters $\mathrm{T}$ and $\mathrm{F}$ as targets and either $\mathrm{E}$ or $\mathrm{O}$ as noise characters.

The significance of our finding of a strong targetbackground similarity effect is that it suggests that some of the theoretical predictions derived by Bjork and Murray may be incorrect (or at least incomplete). We mentioned earlier that the model leads to the prediction that performance would be best in the singletarget condition. Note, however, that this prediction is based upon the assumption that there is a minimal degree of feature overlap between the target letters and the number-sign characters. Otherwise, the input channels leading to detectors associated with these background characters would also be in a heightened state of excitability, and would result in a reduction of target-letter detection. It is for this reason that we consider the crucial finding of Experiment 1 to be that performance in the AA condition was significantly lower than in the $\mathrm{AE}$ and $\mathrm{AK}$ conditions.

\section{EXPERIMENT 3}

The results of Experiment 1 are consistent with the feature-specific inhibition model which postulates that input channels leading to the same feature detectors inhibit one another more than channels leading to different detectors.

We have argued that a response-bias explanation of our data is implausible. However, another possible explanation of the crucial difference between the AA and $\mathrm{AE}$ conditions deserves consideration. This explanation is based on our observation that when two different letters are presented side by side it is relatively easy to determine that two letters have been presented as well as the relative location of each; however, when two closely spaced identical letters are presented briefly, they sometimes appear as one poorly localized letter. So, for example, on some trials when AA is presented, the subject may have the impression that only a single $A$ was present in the display. Furthermore, the subject may be uncertain as to whether or not the A appeared in the cued position. On such trials, a guess would be in order, and the subject, if thinking rationally, would respond "A." However, by assuming that the subject incorrectly guesses $\mathrm{E}$ on a small proportion of the trials, it is possible to account for the obtained data. It is not clear why a subject would ever guess $E$, but it is worthwhile to note that such guessing behavior is not unlike subjects' probability-matching responses obtained in the study of choice behavior (e.g., Humphreys, 1939). (A probability-matching experiment typically requires a subject to guess which of two unequally probable random events will occur on each trial. Over a series of trials, a subject's response probabilities usually come to match the actual probabilities of occurrence of the two events. In a sense, this is "irrational" because a subject could maximize his percentage of correct guesses by always guessing the more frequent event.)

The preceding argument may be considered a specific example of a more general spatial-uncertainty hypothesis. In fact, several investigators have noted that featural information necessary for the identification of a letter may be extracted from a briefly presented visual display independently of the information responsible for determining the letter's spatial location (e.g., Eriksen \& Schultz, 1979; Estes, Allmeyer, \& Reder, 1976; Treisman \& Gelade, 1980; Wolford, 1975). Under such circumstances, a poststimulus cue would not provide much help in resolving any uncertainty about the spatial location of a target letter, since it typically takes a subject $200-300 \mathrm{msec}$ to process a simple visual cue (see, e.g., Eriksen \& Hoffman, 1972, 1973). Undoubtedly, other plausible explanations of the difference between performance in the AA and $\mathrm{AE}$ conditions could be generated based on the premise of spatial uncertainty and the difficulties that arise in the processing of a poststimulus cue.

Experiment 3 was designed to test a number of explanations based on a spatial-uncertainty hypothesis. A new paradigm was employed in which no poststimulus cues were provided. On each trial, the subject was required to indicate by means of a simple "yes"-"no" response whether or not at least one target letter was present in the display. Any effects of spatial uncertainty that may have been present in Bjork and Murray's paradigm were eliminated by no longer requiring subjects to determine the relative location of the target letters in the displays. A spatialuncertainty hypothesis would therefore predict performance to be the same in the AA and AE conditions. However, a lower level of performance in the AA condition would provide further support for the feature-specific inhibition model.

\section{Method}

Subjects. Eight undergraduates at Johns Hopkins University served as paid subjects. None of the subjects had participated in the two previous experiments. All had normal or corrected-to-normal vision.

Apparatus and Procedure. The stimulus materials and procedures were the same as those used in Experiment 1 except for the modifications specified below. The sequence of events occurring on each trial was the same as in Experiment 1, except that the postmask was no longer accompanied by a poststimulus cue. The subject's task was to indicate by means of a "yes"-.no" response whether 
or not at least one of the two target letters, $\mathrm{A}$ or $\mathrm{E}$, was present in a stimulus display. In this new task, information concerning the location of the target letter(s) was not important. If either $A$ or $E$, or both target letters, appeared anywhere in a display, then the correct response was "yes." If neither A nor E was present in a display, then the correct response was "no."

Table 3 lists the four different conditions used in Experiment 3. The "yes" or target-present trials consisted of three double-letter conditions similar to those used in Experiment 1: identical targets (e.g., AA), alternative targets (e.g., AE), single target (e.g., AK). ${ }^{3}$ Note that when two targets are present, as in the identical-and alternative-targets conditions, recognition of either letter is sufficient to make the appropriate response. This leads to the obvious prediction that performance should be greater in these two conditions than in the single-target condition. More importantly, the featurespecific inhibition model predicts less accurate performance in the identical-targets condition than in the alternative-targets condition. The no-target trials comprised displays of the various combinations of the nontarget letters $K$ and $L$.

The target letters $A$ and $E$ appeared equally often in each of the positions of the three target-present conditions. In the single-target condition, the nontarget letters $K$ and $L$ appeared equally often with each of the target letters.

Displays were presented in 20 blocks of 32 trials each. Each block of trials consisted of 16 no-target trials and 16 target-present trials. Eight of the 16 target-present trials consisted of the stimuli from the single-target condition (AK, EK, AL, EL, KA, KE, LA, LE). The remaining eight trials were divided equally among the identicaltargets (AA,EE) and alternative-targets (AE,EA) stimuli. Four of the 16 no-target trials comprised repetitions of a nontarget letter (KK or LL), whereas the remaining 12 trials were divided equally among the two remaining no-target stimuli (KL,LK). Thus, within each block of trials, only half of the eight stimuli that consisted of identical letters (either two of the same target letter or two of the same nontarget) required a "yes" response. This arrangement prevented subjects from basing their "yes"." no" decisions on a general impression of the sameness or difference between the letters presented in the displays.

At the beginning of the experiment, the exposure duration of the stimulus display was adjusted until the subject reached $75 \%$ to $80 \%$ accuracy, averaged across all conditions for four blocks of trials. Durations for the individual subjects ranged from 20 to $55 \mathrm{msec}$, and were considerably shorter than in previous experiments which employed a poststimulus cuing procedure. The exposure duration for each subject was adjusted at the end of each block of trials in order to maintain $75 \%$ to $80 \%$ accuracy throughout the experiment.

Subjects were told at the beginning of the experiment to look for the target letters $A$ and $E$. They were also told to verbally respond "yes" on a trial if either one of the target letters, both of the targets, or two of the same target letters were present. If neither target letter was present in the display, then they were to respond "no." Subjects were fully informed as to the nature of the stimuli, and were shown all the different types of letter pairs along with their appropriate responses prior to experimentation.

\section{Results and Discussion}

Presented in Table 3 are the proportion of correct responses in each display condition. An analysis of the target-present data revealed a significant effect of conditions $[F(2,14)=46.92, p<.001]$. Specifically, performance was lower in the single-target condition than in either the identical-targets condition [t(7) = $3.52, \mathrm{p}<.01]$ or the alternative-targets condition $[\mathrm{t}(7)=$ $9.65, \mathrm{p}<.001]$. This result is not surprising in view of the fact that subjects in the double-target conditions were provided with two opportunities to perceive a target letter, as opposed to just one in the single-target condition.
Table 3

Display Conditions and Mean Proportion of Correct Responses in Each Condition of Experiment 3

\begin{tabular}{ccc}
\hline $\begin{array}{c}\text { Display } \\
\text { Conditions }\end{array}$ & $\begin{array}{c}\text { Sample } \\
\text { Stimulus }\end{array}$ & $\begin{array}{c}\text { Proportion of } \\
\text { Correct }\end{array}$ \\
\hline Targesponses \\
Identical Targets & & \\
Alternative Targets & $\mathrm{AA}$ & .77 \\
Single Target & $\mathrm{AE}$ & .86 \\
No Target Present & $\mathrm{AK}$ & .71 \\
\hline
\end{tabular}

The crucial comparison, however, lies between the two double-target conditions, which showed performance to be significantly lower when the target letters were identical than when they were different $[t(7)=$ $6.87, \mathrm{p}<.001]$. A similar result was obtained within the no-target condition. Performance was lower on no-target trials comprising repetitions of nontarget letters ( $\mathrm{KK}$ and $\mathrm{LL}$ ) than on no-target trials containing two different letters (KL and LK) [t(7) $=2.41, \mathrm{p}<.05]$. That is, subjects were more likely to respond 'yes' when two, identical, nontarget letters were presented than when the nontarget letters were different.

These results are consistent with Bjork and Murray's findings as well as the data obtained in Experiment 1. Furthermore, the fact that the same pattern of results was obtained without the use of poststimulus cues makes any spatial-uncertainty explanation of the difference between performance in the identical-targets (AA) and the alternative-targets (AE) conditions considerably less plausible.

However, these results alone are not conclusive; a similar pattern of results could be predicted based on the assumption that the sensitivity of feature detectors varied independently and randomly from trial to trial. In the alternative-targets condition, for example, two sets of feature detectors are responsive to the targets $\mathrm{A}$ and $\mathrm{E}$, whereas only one set of detectors is responsive to the one type of target letter in the identicaltargets condition. Furthermore, a single set of detectors will more likely be in a state of low sensitivity on any given trial than both sets of the $A$ and $E$ detectors. By way of a specific example, suppose that A detectors are in a state of low sensitivity at a particular point in time (low sensitivity could result from a number of factors such as fatigue). If the letter pair $\mathrm{AE}$ were presented, subjects might not see the A clearly but they would respond correctly that a target was present based on a clear perception of the $\mathrm{E}$. However, if the letter pair AA were presented, then neither instance of the target letter might be seen clearly, resulting in an inaccurate "no" response.

Despite the existence of a possible alternative explanation of the results obtained in Experiment 3, we believe that the data provide converging evidence in favor of the feature-specific inhibition model. In short, the model is supported by the fact that it accurately predicted performance in two different paradigms. 


\section{GENERAL DISCUSSION}

The perceptual interference data reported in this paper can best be accounted for in terms of the featurespecific inhibition model proposed by Bjork and Murray. In this model, which essentially elaborates on Estes' (1972) interactive channels model, two forms of inhibition have been proposed: (1) a general inhibition among input channels going to feature detectors not required for target detection; and (2) a specific inhibition among input channels going to the same feature detector. Moreover, feature detectors are assumed to be of limited capacity (Bjork \& Murray, 1977) and arranged in a hierarchical fashion (cf. Hubel \& Wiesel, 1962, 1965). Inhibition does not imply that one channel prevents the activiation of any other channel, but that the arrival of information over one channel at a common feature detector prevents the simultaneous use of information arriving over the other channels due to the limited capacity of feature detectors (see Pomerantz, Sager, \& Stoever, 1977 , for a similar argument). A delay in the utilization of information arriving over some input channels increases the likelihood that that information will then be lost. This may be particularly evident when the exposure of the stimulus is very brief and is interrupted by a poststimulus mask.

As indicated in the introduction of this paper, evidence in favor of feature-specific inhibition appears to be inconsistent with some previous findings concerning letter perception under tachistoscopic presentation conditions. For example, Eriksen (1966) and Eriksen and Lappin (1965) have shown that target redundancy often facilitates recognition accuracy. These authors found recognition of a target letter to improve as a negatively accelerated function of the number of identical targets in the display. Their data, obtained from both simultaneous and successive presentations of target repetitions, fit a model of perceptual independence. This result appears to conflict with the relatively low performance obtained in the AA condition. However, the amount of separation between letters may be important in resolving this apparent conflict. In the Eriksen studies, the redundant target letters were usually separated by as much as $1.25 \mathrm{deg}$ of visual angle. However, on most trials, Bjork and Murray's letters were separated by less than $1 \mathrm{deg}$ and in our experiments the two letters were contained within $.5 \mathrm{deg}$ of visual angle. These results suggest that perceptual interference between two identical letters may be detectable only when the letters are in close proximity to one another.

It is important to note that in Experiment 3, when subjects. were no longer cued for a particular target position, a redundancy gain was obtained similar to that reported by Eriksen and Lappin (1965). That is, when subjects made the simple judgment of whether or not a target letter was present, performance was better in the identical-targets condition than in the single-target condition. Even though repetition of the same target letter increased performance over that in the single-target condition, it failed to increase performance as much as the addition of the alternative target letter. We believe that this is consistent with other evidence which supports feature-specific interference between identical letters when in close proximity to one another.

Evidence supporting feature-specific interference also appears to be inconsistent with recent evidence exhibiting strong effects of noise letters at the response stage of processing (e.g., Eriksen \& Eriksen, 1974, 1979; Eriksen \& Schultz, 1979). All of these studies employed the same basic paradigm in which subjects respond as quickly and accurately as possible to a centrally located target letter when it is flanked by different types of noise elements. For example, if the target letters $H$ and $K$ were assigned to different responses, then performance could be measured in two types of noise conditions that are relevant to the present discussion: (1) a compatible noise condition (e.g., HHHHHHH), and (2) an incompatible noise condition (e.g., KKKHKKK). In every study reported by Eriksen and his colleagues, it was found that reaction time (RT) to a target letter was longer in the incompatible noise condition than in the compatible noise condition (see also Taylor, 1977).

In order to account for these results, Eriksen and Schultz (1979) proposed a continuous flow model which is based upon the premise that subjects are unable to restrict their visual attention to just the target letter, particularly when the target and noise letters are contained within $1 \mathrm{deg}$ of visual angle (see also Eriksen \& Hoffman, 1972). Such a limitation in selective attention means that as information about the target and noise letters accumulates gradually in the visual system, their respective responses will become activated, requiring the inhibition of responses until the target response reaches threshold. This creates a problem when the target and noise stimuli evoke competing responses, as in our AE displays. Therefore, this model leads to the prediction that performance should be worse in the AE condition than in the AA condition due to response competition between inputs. In the AA condition, the same correct response would be initiated on each trial even if the subject mistakenly processed a noise letter rather than the target letter.

Eriksen and Eriksen (1979) have recently investigated the possibility that the effects of response competition may have obscured or counteracted the effects of perceptual interference in their previous studies. They attempted to reduce the effects of response competition by using noise letters that were from the same response set as the target. For example, with the target letters $\mathrm{H}$ and $\mathrm{S}$ in one response set and the targets $\mathrm{K}$ and $\mathrm{C}$ 
in the other, noise was either identical to the target (e.g., HHHHHHH) or was the other member of the same response set (e.g., SSSHSSS). They reasoned that response competition would be minimized in these two conditions, since the same leverpressing response was required when either $\mathrm{H}$ or $\mathrm{S}$ was the target letter in the middle position. Clearly, the feature-specific inhibition model would predict lower performance when the noise was identical to the target. The results, however, did not support this prediction. Eriksen and Eriksen (1979) argue that although the target and noise elements were from the same response set, a conflict still persisted between internal recognition responses to those stimuli.

In summary, the feature-specific inhibition model proposes that noise letters affect the early perceptual stages of feature extraction, whereas the continuous flow model contends that the effects of noise items are on the response system. We believe that ample evidence has been provided in support of both points of view.

Nevertheless, the question remains: Under what circumstances will evidence be generated in support of feature-specific interference as opposed to response interference? Although a definitive answer is not available at this time, at least one methodological difference between feature-specific and response-interference studies deserves mention. Studies in support of featurespecific interference have employed short-duration data-limited displays (cf. Norman \& Bobrow, 1975), and have used recognition accuracy as the dependent measure of chief interest (Bjork \& Murray, 1977; experiments reported in this paper). However, studies in support of response interference have exposed stimuli for relatively long durations (e.g., $1 \mathrm{sec}$ or longer), and have used RT as the primary dependent measure (e.g., Eriksen \& Eriksen, 1974, 1979; Eriksen \& Schultz, 1979). In fact, Bjork and Murray (1977) found that when both accuracy and RT were measured under data-limited conditions in the same experiment, accuracy was significantly lower in the AA condition (supporting feature-specific interference), whereas $\mathrm{RT}$ was significantly longer in the AE condition (supporting response interference). At first glance, these data suggest that subjects might have adopted different speed-accuracy criteria in the two conditions; however, this explanation is somewhat unreasonable in view of the fact that the conditions appeared randomly from trial to trial.

It appears that a particular dependent measure may play more of a role in determining the experimental outcome than has been previously assumed (see Stanovich, 1979, for a similar argument). Recognition accuracy, which is manipulated by varying stimulus duration, reflects whether or not the information that enters the visual processing system is sufficient to correctly recognize a target letter. The feature-specific inhibition model provides a reasonable account of how the amount of information concerning a target letter can vary under different noise conditions. As noted earlier, the delay and subsequent loss of information arriving over input channels (due to capacity limitations of feature detectors) plays a critical role in determining recognition accuracy when information is no longer coming into the visual system from the stimulus display. Since subjects are not under a time pressure to respond, the effects of competing response tendencies will not be reflected in accuracy measures.

However, when RT is measured stimulus displays are typically presented long enough for target and noise elements to be seen clearly. Under such conditions, target and noise letters may activate competing internal recognition responses (Eriksen \& Eriksen, 1979), and time would be required to inhibit the competing response tendencies. In order to minimize response errors, under the pressure of time subjects are likely to adopt response criteria so high that the effects of perceptual interference among input channels would be essentially undetectable. In other words, if a display is presented long enough for a subject to recognize the target and noise letters, and by that time each has activated its respective response (cf. Eriksen \& Eriksen, 1979), then any further interference among input channels has little or no effect on the accuracy of the subject's response. Bjork and Murray's (1977) results support this point quite nicely. The low level of accuracy in their AA condition relative to the $\mathrm{AE}$ condition indicates that, on the average, less information was available for target recognition in the former condition due to feature-specific inhibition. However, the longer reaction time in the AE condition compared to the AA condition for correct responses, indicates that when enough information is available to recognize the target and noise letters, reaction time is reflective of the processes involved in response interference.

Other potentially important differences exist between studies reporting feature-specific interference and those providing evidence in favor of response interference. For example, the degree of uncertainty regarding a target's location in a visual display may be important. In Eriksen's response-interference studies, a target letter either appeared above the fixation point (e.g., Eriksen \& Eriksen, 1974, 1979; Eriksen \& Schultz, 1979) or was cued by a bar indicator prior to or simultaneous with the onset of a stimulus display (Eriksen \& Hoffman, 1972, 1973). In the feature-specific interference studies, a target letter could appear in two or more locations and was cued after the of fset of a stimulus display. The implication is that feature-specific interference may require a 
subject's attention to be distributed across two or more locations during the stage of feature extraction. This and other methodological differences between feature-specific interference and response-interference studies are currently under investigation.

\section{REFERENCES}

BAmber, D. Reaction times and error rates for "same"'- "different" judgments of multidimensional stimuli. Perception \& Psychophysics, 1969, 6, 169-174.

Bertelson, P. Sequential redundancy and speed in a serial twochoice responding task. Quarterly Journal of Experimental Psychology, 1961, 13, 90-102.

BJork, E. L., \& MURRAY, J. T. On the nature of input channels in visual processing. Psychological Review, 1977, 84, 472-484.

Egeth, H. E., \& BleckeR, D. Differential effects of familiarity on judgments of sameness and difference. Perception \& Psychophysics, 1971, 9, 321-326.

Eriksen, B. A., \& Eriksen, C. W. Effects of noise letters upon the identification of a target letter in a nonsearch task. Perception \& Psychophysics, 1974, 16, 143-149.

ERIKSEN, C. W. Independence of successive inputs and uncorrelated error in visual form perception. Journal of Experimental Psychology, 1966, 72, 26-35.

Eriksen, C. W., \& Eriksen, B. A. Target redundancy in visual search: Do repetitions of the target within the display impair processing? Perception \& Psychophysics, 1979, 26, 195-205.

Eriksen, C. W., \& Hoffman, J. E. Temporal and spatial characteristics of selective encoding from visual displays. Perception \& Psychophysics, 1972, 12, 201-204.

Eriksen, C. W., \& Hoffman, J. E. The extent of processing of noise elements during selective encoding from visual displays. Perception \& Psychophysics, 1973, 14, 155-160.

Eriksen, C. W., \& Lappin, J. S. Internal perceptual system noise and redundancy in simultaneous inputs in form identification. Psychonomic Science, 1965, 2, 351-352.

Eriksen, C. W., \& Schultz, D. W. Information processing in visual search: A continuous flow conception and experimental results. Perception \& Psychophysics, 1979, 25, 249-263.

Estes, W. K. Interactions of signal and background variables in visual processing. Perception \& Psychophysics, 1972, 12, 278-286.

EstEs, W. K. Redundancy of noise elements and signals in visual detection of letters. Perception \& Psychophysics, 1974, 16, 53-60.

Estes, W. K., Allmeyer, D. H., \& Reder, S. M. Serial position functions for letter identification at brief and extended exposure durations. Perception \& Psychophysics, 1976, 19, $1-15$.

Felfoldy, G. L. Repetition effects in choice reaction time to multidimensional stimuli. Perception \& Psychophysics, 1974, $15,453-459$.

Gardner, G. T. Evidence for independent parallel channels in tachistoscopic perception. Cognitive Psychology, 1973, 4, 130-155.

Hubel, D. H., \& Wiesel, T. N. Receptive fields, binocular interaction and functional architecture in the cat's visual cortex. Journal of Physiology, 1962, 160, 106-154.

HubeL, D. H., \& WiEsEL, T. N. Receptive fields and functional architecture in two nonstriate visual areas (18 and 19) of the cat. Journal of Neurophysiology, 1965, 28, 229-289.
Humphreys, L. G. Acquisition and extinction of verbal expectations in a situation analogous to conditioning. Journal of Experimental Psychology, 1939, 25, 294-301.

Kinchla, R. A. Detecting target elements in multielement arrays. Perception \& Psychophysics, 1974, 15, 149-158.

Krueger, L. E. A theory of perceptual matching. Psychological Review, 1978, 85, 278-304.

McIntyre, C., Fox, R., \& Neale, J. Effects of noise similarity and redundancy on the information processed from brief visual displays. Perception \& Psychophysics, 1970, 7, 328-332.

Norman, D. A., \& Boв row, D. G. On data-limited and resourcelimited processes. Cognitive Psychology, 1975, 7, 44-64.

Pomerantz, J. R., Sager, L. C., \& Stoever, R. J. Perception of wholes and of their component parts: Some configural superiority effects. Journal of Experimental Psychology: Human Perception and Performance, 1977, 3, 422-435.

Rumelhart, D. E. A multicomponent theory of the perception of briefly exposed visual displays. Journal of Mathematical Psychology, 1970, 7, 191-218.

Schyaneveldt, R,, \& Meyer, D. E. Retrieval and comparison processes in semantic memory. In S. Kornblum (Ed.), Attention and performance IV. New York: Academic Press, 1973.

Shiffrin, R. M., \& Geisler, W. S. Visual recognition in a theory of information processing. In R. L. Solso (Ed.), Contemporary issues in cognitive psychology: The Loyola Sym. posium. Washington, D.C: Winston, 1973.

Stanovich, K. E. Studies of letter identification using qualitative error analysis: Effects of speed stress, tachistoscopic presentation, and word context. Journal of Experimental Psychology: Human Perception and Performance, 1979, 5, 713-733.

TAYLOR, D. A. Time course of context effects. Journal of Experimental Psychology: General, 1977, 106, 404-426.

TownsEND, J. T. Theoretical analysis of an alphabetic confusion matrix. Perception \& Psychophysics, 1971, 9, 40-50.

Treisman, A. M., \& Gelade, G. A feature-integration theory of attention. Cognitive Psychology, 1980, 12, 97-136.

Wolford, G. Perturbation model for letter identification. Psychological Review, 1975, 82, 184-199.

\section{NOTES}

1. This response system eliminated the left-right cue-response compatibility effects that were present in a pilot investigation. Requiring verbal responses helped subjects map the two letters to the appropriate manual responses. Whenever a discrepancy between a manual and a verbal response was obtained, the latter was counted as being the intended response. This situation arose in less than $1 \%$ of the trials, and in most cases subjects spontaneously corrected themselves by indicating which was the intended response.

2. Since the experiments reported in this paper were designed to test the major predictions of the feature-specific inhibition model, all multiple comparisons were planned.

3. In the context of Experiment 3, it is appropriate to refer to a stimulus such as AK as a single target display. Note, however, that in Experiment 1 such displays were called noise nontarget displays. (The A\# stimuli used in Experiments 1 and 2 were not used in Experiment 3.)

(Received for publication October 25, 1979; revision accepted February 11,1980 .) 